\title{
The Research of Heat change in the process of bath Wenjun Cao
}

\author{
School of North China Electric Power University Baoding, Baoding 071000, China; \\ 916827591@qq.com
}

Keywords: Heat transfer Differential Equation Newton's law of cooling

\begin{abstract}
Based on Newton's law of cooling and convection heat transfer theory, we analyze the temperature change in space, we divide the heat transfer in the process of bathinto four parts and give the expression of each part, then we obtain an ordinary differential equation about the temperature of water. We can solve the equation under different rules to research the heat problem.

As Figure 1 shows, the heat transfer in the process of bath can be divided into four parts:
\end{abstract}

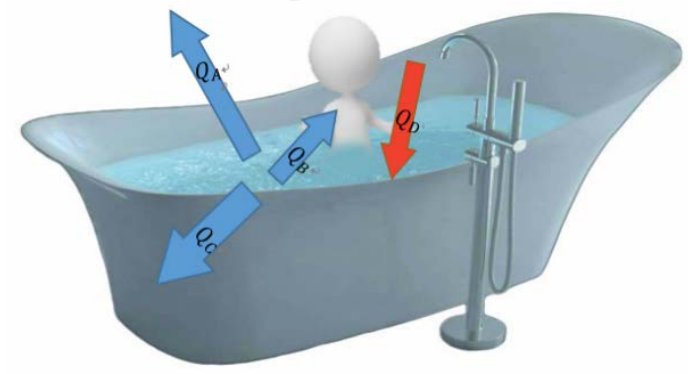

Figure 1. the heat transfer in the process of bath

Step 1. Heat dissipation from water tosurrounding air (): There are three forms of hot water to dissipation heat to the surrounding air [1]:

- Convection: Let denotes the heat transfer from water with the area to surrounding air within the time $d t$. The convection results in the decrease of water temperature in the bathtub, so we consider that is a negative number. can be represented using the following formula:, where $\alpha$ is the heat release coefficient of water and air.

- Evaporation: stands for the heat of water evaporation with the area and the time $d t$. It is also a negative number and can be represented as:,where is the evaporation coefficient of water, is the steam pressure of saturated layer on the water surface and is the partial steam pressure of moist air.

- Radiation:Weregardas the radiation heat(within and $d t$ ), based on Stefan-Boltzmann law, we have:.is emissivity and is Stefan-Boltzmann constant of 5.6.

Based on our analysis above, we put forward the expression of the total heat dissipation from water to the surrounding air:

Step 2.Heat dissipation from water to the person in the bath tub(): According to our assumption that the temperature of human body is constant, the heat dissipation only consists of one part: heat transfer from water to human body. The heat transfer process between water and person in the bathtub is mainly convective heat transfer. Therefore according to Newton's law of cooling, we have the expression of heat transfer with the area (let be the area of contact part of the water and human body) and the time $d t$ :

In this formula, his the convective heat transfer coefficient between water and human body. The negative sign means the water in the bathtub loses heat.

Step 3. Heat dissipation from water to outside through bath tub wall ():The temperature difference between water in the bathtub and surrounding air can't be neglected. In Step 1, we have calculated the heat dissipation on the contact area of water and air. So now we consider the overall heat transfer process through bathtub wall. 
We only consider the steady state and regard air as cool fluid. In general, overall heat transfer process contains three parts [2]:

- Heat transfer from hot fluid to high temperature side of bathtub wall.

- Heat transfer from the high temperature side to the low temperature side of bathtub wall.

- Heat transfer from the low temperature side of bathtub wall to the cool fluid.

All three periods are in series. Since it is steady state process, the heat transfer in each period is the same, represented by .

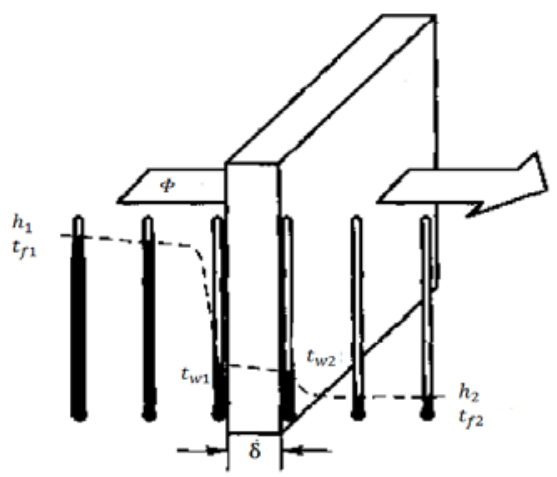

Figure 2. Overall heat transfer process through bathtub wall

Let denote the area of bathtub wall, according to Figure 2, we have the expression of heat transfer of three periods:, ,.

We rewrite three formulas above for simplicity:, .Add three formulas above and eliminate the temperature , we have:, where is the temperature of hot fluid which is also - the temperature of water. And is also .

So based on what we have derived, we have the expression of heat dissipation from water to outside through the bathtub with the area and the time $d t$. We call it, like our previous analysis, it is also a negative number.

In this formula,is the thermal conductivity of bathtub wall. is the convective heat transfer coefficient between water and bathtub wall and is the convective heat transfer coefficient between surrounding air and bathtub wall. is the thickness of bathtub wall and it is determined by the shape and volume of tub.

Step 4. Heat changes because of the inflow and outflow of water (): The person in tub adds a constant trickle of hot water which can bring heat. And excess water escapes through an overflow drain which will take away heat. We use to represent the change of heat because of the update of water in tub.

We define the change of heat is when the volume $d V$ ofwater changes. Since we assume that the volume of the water in bathtub is constant, we can consider the volume $d V$ of hot water is added into bathtub while the volume $d V$ of water escapes. Hence we have:

We use u to denote the velocity of hot water from faucet as well as the velocity of outflow water, that is to say:.So we can get the expression of :

Step.5 Ordinary differential equation: Basedon our analysis above, the total change of heat consists of four parts. The total heat change of water in tub is:.For the calculation in next step, we change the formula above into:

We substitute (1) (4) into formula (5) and have:

For the calculation formula of specific heat capacity: 
is the initial temperature of water in tub. We differentiate both sides of formula (7) at the same time and get:

Then we can get an ordinary differential equation for the simultaneous equations of (6) and (8).

\section{Summary}

Through the analyses above, we get an ordinary differential equation to describe the dynamic change in the process of bath. This formula can be used for calculation of related problems. The formula is showed below:

\section{References}

[1] ZHAO Zhen-guo. Enthalpy difference formula for calculating heat dissipation on water surface and its use [J]. Journal of Hydraulic Engineering, 2004(2): 34-38.

[2]YANG Shi-ming, TAO Wen-quan. Heat Transfer, $4^{\text {th }}$ ed, Beijing: Higher Education Press, 2006. 